\title{
Cuidado sensible y seguridad del apego en preescolares
}

\section{Sensitive caregiving and attachment security in preschoolers}

\author{
Elena Díaz Mosquera ${ }^{1}$ \\ Ivonne Andrade Zúñiga ${ }^{2}$ \\ Elisa Espinosa Marroquín ${ }^{3}$ \\ Magaly Nóblega ${ }^{4}$ \\ Juan Núñez del Prado \\ ${ }^{1,2,3}$ Facultad de Psicología, Pontificia Universidad Católica del Ecuador \\ ${ }^{4,5}$ Departamento de Psicología, Pontificia Universidad Católica del Perú
}

\begin{abstract}
Resumen: Este estudio se fundamentó en la hipótesis de sensibilidad de la teoría del apego que relaciona la crianza sensible con la seguridad del apego. Tuvo como objetivo analizar la relación entre la sensibilidad de 16 figuras cuidadoras y la seguridad del apego de sus hijos preescolares de desarrollo típico, residentes en Quito, Ecuador. Para la medición se utilizaron dos instrumentos: Maternal Behavior for Preschooler Q-set y Attachment Behavior Q-set Version 3.0, en su adaptación latinoamericana. En comparación con otros estudios con población latina, en este trabajo se encontró un nivel de sensibilidad similar al previamente reportado y un nivel de seguridad ligeramente más alto. En cuanto a la relación entre ambas variables, se halló a la sensibilidad como predictora de la seguridad del apego. Al ser Ecuador un país pluricultural, se recomienda replicar este estudio en grupos de otros contextos culturales y con otras condiciones de desarrollo.
\end{abstract}

Palabras clave: cuidado infantil, relaciones de apego, niños en edad preescolar, desarrollo socioemocional

\begin{abstract}
This study was based on sensitivity hypothesis of attachment theory that relates sensitive nurture with attachment security. The aim was to analyze the relation between 16 caregivers' sensitivity and attachment security of their preschool children of typical development, living in Quito, Ecuador. Two instruments were used for assessment: Maternal Behavior for Preschoolers Q-set and Attachment Behavior Q-set Version 3.0, in their Latin American adaptation. Compared to others studies with Latino population, in this work a level of sensitivity similar to the previously reported, and a slightly higher level of children security was found. Regarding the relation between both variables, sensitivity was found as a predictor of attachment security. Since Ecuador is a multicultural country, is recommended to replicate this study in other cultural contexts and with other development conditions groups.
\end{abstract}

Key words: children care, attachment relations, preschool children, socio-emotional development

Cómo citar este artículo:

Díaz Mosquera, E., Andrade Zúñiga, I., Espinosa Marroquín, E., Nóblega, M., \& Núñez del Prado, J. (2018). Cuidado sensible y seguridad del apego en preescolares. Ciencias Psicológicas, 12(1), 97-107. doi: https://doi.org/10.22235/cp.v12i1.1600

Correspondencia: Elena Díaz Mosquera, Ivonne Andrade Zúñiga, Elisa Espinosa Marroquín, Facultad de Psicología Pontificia Universidad Católica del Ecuador. Av. 12 de Octubre 1076 y Roca, Apartado Postal 17012184, Quito, Ecuador. Magaly Nóblega, Juan Núñez del Prado, Departamento de Psicología, Pontificia Universidad Católica del Perú. Av. Universitaria 1801, San Miguel, Lima, Perú, e-mail: endiaz@puce.edu.ec, iandrade@puce.edu.ec,3aespinosa704@puce.edu.ec.,mnoblega@pucp.pe, j.nunezdelprado@pucp.pe 


\section{Introducción}

Los estudios sobre el apego realizados por Bowlby entre los años 1960 y 1980 se han constituido en sólidos constructos teóricos sobre el desarrollo socioemocional. Según sus planteamientos, los infantes disponen de un sistema conductual de apego que los conduce a vincularse de manera preferente con una figura que tiene el rol de cuidador principal (Bowlby, 1969/1998; 1988; Bakermans-Kranenburg \& van Ijzendoorn, 2016). Cuando este vínculo es seguro, se presenta en el niño un balance apropiado entre búsqueda de proximidad con el cuidador y exploración del ambiente (Ainsworth, 1969; Bowlby, 1969/1998; 1988), balance en el cual la figura cuidadora se constituye en una base segura que ofrece oportunidades de aprendizaje mediante interacciones exploratorias, con su supervisión y apoyo (e.g., Salinas-Quiroz et al., 2015; Simpson \& Belsky, 2016).

Por lo tanto, la seguridad del apego se define como la confianza que experimenta el niño en que una persona cercana a él, su figura de apego, está disponible, le ofrece consuelo y protección y es responsiva a sus señales de estrés o a su solicitud de contacto (Bowlby, 1988; BakermansKranenburg \& van Ijzendoorn, 2016). Investigaciones recientes han encontrado que el apego favorece el desarrollo de las estructuras cerebrales relacionadas con la vida emocional y social (e.g., Coan, 2016; Schore, 2017), por lo que se considera que la calidad de este vínculo es un antecedente del estado de bienestar y del sentido implícito de seguridad a lo largo de la vida (Schore, 2017; Simpson \& Belsky, 2016).

En referencia a los postulados de Bowlby y como resultado de sus observaciones, Ainsworth $(1967 ; 1969)$ encontró que la sensibilidad de las figuras cuidadoras hacia las señales de los infantes antecede a la seguridad del apego y es su determinante primario (Ainsworth, Blehar, Waters, \& Wall, 1978/2015; Pederson, Bailey, Tarabulsy, Bento, \& Moran, 2014; Salinas-Quiroz \& Posada, 2015). La sensibilidad se refiere a la habilidad del cuidador para percibir e identificar las señales y comunicaciones implícitas en la conducta del niño, interpretarlas de manera adecuada y responder a ellas apropiadamente y con prontitud (Ainsworth, 1969). En consecuencia, el nivel de sensibilidad de la figura cuidadora se manifiesta en la calidad de sus respuestas a las necesidades cambiantes y a veces ambiguas del niño (Cerezo, Pons-Salvador, \& Trenado, 2011).

Los hallazgos de la amplia variedad de investigaciones sobre el tema realizadas especialmente en Europa y Norteamérica (e.g., De Wolf \& van Ijzendoorn, 1997; Mesman \& Emmen, 2013), llevaron al planteamiento de las cuatro hipótesis centrales de la teoría del apego: universalidad, normatividad, sensibilidad y competencia (Mesman, van IJzendoorn, \& Sagi-Schwartz, 2016; van IJzendoorn \& Sagi-Schwartz, 2008). En relación a este último aspecto, la competencia, varios estudios han fundamentado el efecto de los vínculos tempranos de apego en el desarrollo cognitivo y social que se evidencia a partir de la etapa preescolar (e.g., Marvin, Britner, \& Rusell, 2016).

En Latinoamérica, la investigación sobre sensibilidad - seguridad en la infancia y en la niñez es más reciente y menos prolífera, a pesar de que Bowlby (1988) y varios autores contemporáneos (e.g., Carbonell, 2013; Keller, 2013; Quinn \& Mageo, 2013; Salinas-Quiroz \& Posada, 2015) han destacado la importancia de realizar estudios en ambientes ecológicos diversos, con la finalidad de identificar los determinantes culturales propios de cada contexto, fomentar la implementación de intervenciones basadas en evidencias (Carbonell, 2013), y validar en otros espacios las hipótesis centrales de la teoría del apego.

Básicamente, las publicaciones de estudios latinoamericanos sobre el tema se han realizado con población de Chile (e.g., Lecannelier, Kimelman, González, Núñez, \& Hoffman, 2008), Colombia (e.g., Posada, Carbonell, Alzate, \& Plata, 2004; Posada et al., 2002; Vaughn et al., 2007), Perú (e.g., Nóblega, 2012; Nóblega et al., 2016), México (e.g., Posada et al., 2013). Los resultados generales de varios de estos estudios han demostrado asociaciones significativas entre sensibilidad y seguridad, lo cual pone en evidencia el rol fundamental del cuidado sensible en la organización de la conducta de base segura. Adicionalmente, se ha encontrado que la edad, la educación y las condiciones de vida de la figura cuidadora están positivamente relacionadas con su nivel de sensibilidad (Posada et al., 2016; Santelices et al., 2015).

En el caso de Ecuador, las publicaciones sobre relaciones de apego en la niñez son escasas, aunque existen estudios que sustentan algunas asociaciones, por ejemplo, rasgos de persona- 
lidad de la madre y tipo de apego de los hijos (Guerrero, 2013), estilo de apego infantil, adaptación escolar y aprendizaje (Orbe, 2012; 2015). Sin embargo, una revisión teórica de varios trabajos universitarios sobre el tema, cuestiona los criterios normativos de la teoría del apego en sociedades que poseen diversidad de modelos culturales, como la ecuatoriana (Villamarín, 2017). Se plantea, de este modo, una problemática: la necesidad de caracterizar de manera contextualizada, tanto el comportamiento sensible de las figuras cuidadoras, como las manifestaciones de la seguridad del apego de sus hijos.

En articulación con lo expuesto, este estudio se fundamentó en la hipótesis de sensibilidad de la teoría del apego que relaciona crianza sensible con seguridad (van IJzendoorn \& Sagi-Schwartz, 2008), mediante un enfoque mixto de investigación. El objetivo fue analizar la relación entre la sensibilidad de un grupo de figuras cuidadoras y la seguridad del apego de sus hijos preescolares de desarrollo típico, residentes en Quito, Ecuador, con la finalidad de generar conocimiento contextual y contar con datos referenciales que sirvan de base para trabajar con otros grupos culturales y con población que presenta capacidades diferentes.

Los lineamientos de este trabajo, así como el Protocolo de Consentimiento Informado para Participantes que se empleó, fueron aprobados por el Comité de Ética de la Investigación con Seres Humanos de la Pontificia Universidad Católica del Ecuador (PUCE). En el diseño de la investigación, se replicó parcialmente el modelo de un estudio realizado por el Grupo de relaciones vinculares y desarrollo socioemocional de la Pontificia Universidad Católica del Perú (PUCP).

\section{Materiales y métodos}

\section{Participantes}

Los participantes en este estudio fueron 16 díadas de figuras cuidadoras y sus hijos de desarrollo típico en edad preescolar, de nivel socio económico medio, residentes en Quito, Ecuador. Como criterios de inclusión para las figuras cuidadoras se estableció que fueran adultas y que tuvieran, como mínimo, educación secundaria completa.

Para reclutar a los participantes, la información sobre el estudio fue difundida a través de diversos medios: una charla en una unidad edu- cativa de la localidad, avisos en las carteleras de un centro pediátrico y de un centro infantil, y publicaciones en redes sociales. Con las personas que manifestaron su interés en participar y que, al mismo tiempo, reunían los criterios de inclusión, se suscribió un Consentimiento Informado en el que las figuras adultas, como guardas legales, autorizaban la participación de sus niños. Adicionalmente, a través de una Entrevista inicial se recabó información sociodemográfica y datos sobre el desarrollo del niño. Por lo tanto, la selección de participantes se realizó por conveniencia. El proceso se inició con 22 díadas, de las cuales únicamente 16 lo culminaron.

En todos los casos, las madres se auto identificaron como las figuras cuidadoras principales de los niños. Su edad fluctuaba entre 22 y 44 años $(M=33.81, D E=5.62)$. En relación a su nivel de instrucción, el promedio de años de estudio fue de $15.63($ Min=12, Max=18, $D E=.96)$, así, tres de ellas (19\%) tenían secundaria completa, 10 (62\%) tenían educación superior de pregrado, y las otras tres $(19 \%)$ contaban con estudios superiores a nivel de posgrado. En cuanto al número de hijos de la madre, ocho de ellas (50\%) tenían uno solo, tres $(18.75 \%)$ tenían dos hijos, y cinco de ellas $(31,25 \%)$, tres hijos.

Los niños tenían entre 36 y 71 meses de edad $(M=56.94, D E=7.91)$; ocho de ellos $(50 \%)$ eran varones y ocho (50\%), mujeres. En cuanto a su posición ordinal, ocho eran hijos únicos $(50 \%)$, cuatro eran los hijos mayores (25\%), dos eran los intermedios (12.5\%), y dos eran los menores $(12.5 \%)$ entre sus hermanos. A través de la Entrevista inicial con las figuras cuidadoras, se verificó que todos los niños habían tenido un desarrollo bio-psico-social dentro de la norma.

Al finalizar el proceso, como beneficio por su participación, se ofreció a las figuras cuidadoras recomendaciones para fortalecer los vínculos de apego con sus hijos.

\section{Medición}

Cuidado sensible. La sensibilidad del cuidado fue evaluada con la escala Maternal Behavior for Preschoolers Q-Set (MBPQS) en su versión actualizada (Posada, Kaloustian, Richmond, \& Moreno, 2007), adaptada lingüísticamente a las expresiones idiomáticas de Perú (Nóblega, 2012), que son muy similares a las que se emplean en Ecuador. MBPQS consta de 90 enun- 
ciados que describen conductas vinculadas al constructo de sensibilidad, 55 de los cuales se refieren a cuatro dimensiones: Contribución a interacciones armoniosas (CIA), Apoyo a la base segura (ABS), Supervisión y monitoreo (SUP), y Establecimiento de límites (EL).

Según Posada y sus colaboradores (2007), para la administración de la escala dos evaluadores observan de manera independiente la conducta de la figura cuidadora en su interacción con el niño en el ambiente natural de ambos y aplican, a manera de entrevista, los ítems de la escala que no son fácilmente observables (e. g., Ítem 67: "Cuando establece reglas y prohibiciones al niño en una actividad, le explica las razones"). Luego de ello, los evaluadores utilizan la metodología Q-sort para evaluar la conducta observada, para lo cual clasifican los 90 enunciados en grupos que van en un rango de 9 a 1 con 10 enunciados en cada uno, de modo que en el grupo 9 se ubican las conductas más características de la figura evaluada, y en el 1, las menos características. Posteriormente, se calcula el promedio de los puntajes si la diferencia entre ambos observadores es de hasta tres puntos; si la diferencia es mayor, tienen que revisar la conducta y llegar a un acuerdo. El puntaje final de cada ítem es correlacionado con el criterio de la escala, para obtener el índice de sensibilidad de la figura evaluada (Posada et al., 2007).

En la literatura se reportan dos estudios en los que se ha encontrado soporte empírico de la confiabilidad y validez de MBPQS y de sus cuatro dimensiones (Posada et al., 2007; Richmond, Posada, \& Jacobs, 2001). Respecto de la confiabilidad interobservador, estudios transculturales recientes la ubican en un rango entre .81 (Perú) y .86 (Estados Unidos) (Posada et al., 2016). En cuanto a la confiabilidad por consistencia interna, los mismos estudios transculturales reportan un alfa de Cronbach entre .93 (CIA) y .67 (EL) en la muestra total (Posada et al., 2016).

En el presente estudio, se grabaron las conductas de las 16 figuras cuidadoras en la interacción habitual con sus niños, en el ambiente natural de ambos (su hogar), por espacio de una hora. Luego de ello, dos observadores independientes previamente entrenados en el uso de la escala MBPQS, evaluaron las conductas. La confiabilidad interobservador promedio encontrada fue de .86 ( $D E=0.07$, Min=.73, Max=.95), la cual fue calculada con el coeficiente de correlación intra- clase. En la confiabilidad por consistencia interna de las cuatro dimensiones de la sensibilidad de MBPQS, se encontraron coeficientes altos, similares a los reportados por Posada y sus colaboradores (2016): $\alpha=.96$ en CIA, $\alpha=.95$ en ABS, $\alpha$ $=.71$ en SUP, $\mathrm{y} \alpha=.81$ en EL.

Seguridad del apego. La conducta de apego de los niños participantes fue evaluada con la escala Attachment Behavior Q-Set, Versión 3.0 (AQS) (Waters, 1995), adaptada al contexto latinoamericano (Posada, Waters, Crowell, \& Lay, 1995) y a las expresiones lingüísticas de Perú (Nóblega, 2012). Al igual que la escala anterior, AQS consta de 90 enunciados que evalúan, en este caso, conductas relacionadas con la seguridad del apego; 50 de los enunciados se refieren a cuatro dimensiones: Calidez de las interacciones con la madre (CIM), Placer en el contacto físico con la madre (PCM), Interacción del niño con otros adultos (IOA), y Búsqueda de proximidad con la madre (BPM).

De la misma manera que la escala anterior, dos evaluadores observan de forma independiente la conducta de interacción del niño con su figura cuidadora en el ambiente natural de ambos y aplican a la figura adulta los ítems de la escala AQS que no son fácilmente observables (e. g., Ítem 3: "Cuando está molesto o enfermo, el niño acepta que otros adultos diferentes a su figura cuidadora, lo consuelen"). Luego de ello, los evaluadores utilizan la metodología Q-sort previamente descrita, para calificar la conducta observada. El índice de seguridad se obtiene mediante la correlación de los puntajes finales de cada ítem con el criterio de la escala (Waters, 1995).

En la literatura se reportan varios estudios en los que se ha documentado la confiabilidad y validez de AQS y de sus cuatro dimensiones en contextos diferentes a los clásicos (e.g., Posada et al., 1995; Posada et al., 2013; Solomon \& George, 2016; van IJzendoorn, Vereijken, Bakermans-Kranenburg, \& Riksen-Walraven, 2004). En referencia a la confiabilidad interobservador, los estudios transculturales conducidos por Posada et al. (2016) la ubican en un rango entre .78 (Estados Unidos) y .84 (Colombia). Respecto de la confiabilidad por consistencia interna, se ha señalado un $\alpha>.85$ en la escala total (Vaughn et al., 2007), en tanto que en las dimensiones de la seguridad del apego, el trabajo conducido por Nóblega y sus colaboradores (2016) reporta un $\alpha$ entre .60 en PCM e IOA, y .89 en CIM. 
En el presente estudio, la escala AQS y la escala MBPQS fueron aplicadas simultáneamente. En el caso de AQS, se grabó la conducta de los 16 niños en la interacción habitual con sus figuras cuidadoras, en el ambiente natural de ambos (su hogar), por espacio de una hora, luego de lo cual, dos observadores independientes previamente entrenados, evaluaron la conducta de los niños. La confiabilidad interobservador promedio encontrada fue de $.87(D E=0.06, \mathrm{Min}=.76$, Max=.98), obtenida con el coeficiente de correlación intraclase. La confiabilidad por consistencia interna de las cuatro dimensiones de la seguridad de AQS, calculada con el alfa de Cronbach, fue de $\alpha=.82$ en CIM, $\alpha=.696$ en PCM, $\alpha=.760$ en BPM, y $\alpha=.847$ en IOA.

\section{Procedimiento}

Se realizaron cuatro visitas al hogar de cada díada. En la primera visita se informó a las figuras cuidadoras sobre los objetivos, procedimientos, su rol en el estudio; se solventaron dudas y se suscribió el consentimiento informado, documento en el cual confirmaban su voluntad de participar, autorizaban la participación de sus hijos y aceptaban el uso de los datos con fines investigativos. En la segunda visita se aplicó la entrevista inicial para recabar información sociodemográfica y obtener datos sobre el desarrollo del niño. En la tercera visita se observó y grabó la interacción diádica habitual en su ambiente natural, con una duración aproximada de una hora, luego de lo cual se aplicaron a las figuras cuidadoras las preguntas de entrevista de algunos de los ítems de MBPQS y AQS que no son fácilmente observables. En una última visita, se brindaron recomendaciones para fortalecer el vínculo de apego con los niños, en un contexto conversacional.

Cada filmación de la relación diádica de los 16 participantes fue observada por cuatro observadores independientes previamente capacitados en los instrumentos; dos de ellos evaluaron la conducta materna con la escala MBPQS y los otros dos, la conducta del niño con la escala AQS; en todos los casos se utilizó la metodología Q-sort.

Para el análisis de los datos se utilizó estadística descriptiva (media, desviación estándar, puntajes mínimos y puntajes máximos), estadística paramétrica, básicamente, correlación de Pearson, y estadística inferencial, fundamen- talmente regresión lineal. Estos procedimientos permitieron analizar las características de la sensibilidad de las madres y de la seguridad de los niños, así como la asociación entre sensibilidad y seguridad.

\section{Resultados}

Respecto de la sensibilidad de las 16 figuras cuidadoras, se encontró un índice promedio de $.43(D E=0.44$, Min=-.63, Max $=.77$, IC 95\%= $[.20, .67])$ entre los puntajes promedio de las participantes y el criterio de sensibilidad de la escala MBPQS, índice que se ubica dentro de los rangos encontrados en otros países latinoamericanos (e.g., Posada et al., 2016). En cuanto a la consistencia de la conducta de la figura cuidadora, se hallaron correlaciones altas y muy altas entre la sensibilidad y sus cuatro dimensiones, y entre las dimensiones entre sí, como se puede observar en la Tabla 1; estas correlaciones son, además, estadísticamente significativas a un nivel de $p<.01$; la asociación más baja se registra entre Establecimiento de límites y Supervisión y monitoreo $(r=.53)$.

Tabla 1

Correlación entre sensibilidad materna y sus dimensiones

\begin{tabular}{lccccc}
\hline & Sensibilidad & CIA & ABS & SUP & EL \\
\hline Sensibilidad & - & & & & \\
CIA & $.98^{* *}$ & - & & & \\
ABS & $.97 * *$ & $.96 * *$ & - & & \\
SUP & $.90 * *$ & $.90 * *$ & $.88^{* *}$ & - & \\
EL & $.80 * *$ & $.75 * *$ & $.76^{* *}$ & $.53 * *$ & - \\
& & & & & \\
\hline
\end{tabular}

Nota $:$ CIA = Contribución a interacciones armoniosas; ABS = Apoyo a la base segura; SUP = Supervisión y monitoreo; EL = Establecimiento de límites.

$* p<.05 ; * * p<.01$

El análisis de la sensibilidad en relación con los datos sociodemográficos, evidenció una correlación positiva moderada y significativa estadísticamente entre cuidado sensible y edad de la figura cuidadora $(r=.526$, IC $95 \%=[.04, .81]$, $p=.036$ ), hallazgo que coincide con reportes previos (Posada et al., 2016; Santelices et al., 2015); respecto de la asociación entre cuidado sensible e instrucción de la figura cuidadora, se halló una correlación positiva y moderada, pero no significativa a nivel estadístico $(r=.423, I C 95 \%=[-.09$, .76], $p=.102$ ). 
En referencia a las puntuaciones medias obtenidas con la aplicación de MBPQS, se encontraron discrepancias con el criterio de la escala mayores $\mathrm{a}+-3$ puntos en seis de los 90 ítems, como se aprecia en la Tabla 2.

Según se puede observar, dos de los seis ítems, pertenecientes a la dimensión de Apoyo a la base segura, recibieron un puntaje mayor que el de la escala, en tanto que los otros tres ítems (uno de Apoyo a la base segura y dos de Supervisión y monitoreo), recibieron un puntaje menor. El análisis del enunciado de estos ítems permite inferir que en el grupo de figuras cuidadoras participantes existe, en general, una disposición mayor a la normada en la escala para realizar actividades en base a lo que le llama la atención al niño, mientras que por otro lado, presentan, de manera general, una tendencia menor a la del criterio para calmar al niño y prevenir situaciones conflictivas.

Respecto de la seguridad del apego de los niños, se encontró un índice medio de $.42(D E=0.17$, Min $=.02$, Max $=.71$, IC 95\% = [.33, .51]), que es un poco más alto que el hallado en otros países latinoamericanos (Posada et al., 2016). En cuanto a la consistencia de la seguridad del apego (Tabla 3) se encontró que, en los niños participantes, la seguridad está asociada a nivel alto y significativo con la calidez en las interacciones con la madre $(p<.01)$, a nivel moderado y significativo con el placer que obtiene en el contacto físico con ella $(p<.05)$, y a nivel moderado con la búsqueda de su proximidad; se halló también que la seguridad del apego es independiente de la interacción que el niño establece con otros adultos, y que existe una asociación entre baja y nula de las dimensiones de la seguridad del apego entre sí.

Por otro lado, las correlaciones encontradas entre seguridad del apego y algunos datos sociodemográficos como edad de los niños $(r=.167$, IC 95\% $=[-.353, .582], p=.537)$, y entre seguridad del apego y género $(r=.138$, IC 95\% $=[-.395$, $.588], p=.609$ ), fueron bajas y no significativas a nivel estadístico. En referencia a la Media de las puntuaciones en la escala AQS (Tabla 4), se encontraron discrepancias con el criterio mayores a +-3 puntos en 11 de los 90 ítems.

Cinco de estos 11 ítems (dos de la dimensión de Búsqueda de la proximidad de la madre, uno de la dimensión de Interacción con otros adultos, y dos que no pertenecen a ninguna de las
Tabla 2

Discrepancias mayores $a+-3$ puntos en MBPQS $(N=16)$

\begin{tabular}{|c|c|c|c|c|c|}
\hline $\begin{array}{l}\text { Ítem } \\
\mathrm{N}^{\circ}\end{array}$ & Dim & Enunciado & $\begin{array}{l}\text { Ptje } \\
\text { Medio }\end{array}$ & $\begin{array}{c}\text { Ptje } \\
\text { Criterio }\end{array}$ & Discr \\
\hline 57 & ABS & $\begin{array}{l}\text { Cuando el niño está } \\
\text { molesto o triste, lo } \\
\text { ignora o no es muy } \\
\text { hábil calmándolo } \\
\text { y regresándolo de } \\
\text { nuevo al juego. }\end{array}$ & 5.06 & 1 & -4.06 \\
\hline 36 & ABS & $\begin{array}{l}\text { Realiza actividades } \\
\text { basándose en lo que } \\
\text { le llama la atención } \\
\text { al niño. }\end{array}$ & 6.75 & 3 & -3.75 \\
\hline 40 & SUP & $\begin{array}{l}\text { Está dos pasos } \\
\text { adelante } \\
\text { niño; del anticipa } \\
\text { las potenciales } \\
\text { s i t u a c i o n e s } \\
\text { conflictivas } \\
\text { hace cosas para } \\
\text { prevenirlas. }\end{array}$ & 5.03 & 8.5 & 3.47 \\
\hline 55 & ABS & $\begin{array}{l}\text { Cuando ocurre } \\
\text { un accidente, } \\
\text { inmediatamente va } \\
\text { hasta donde está el } \\
\text { niño para revisar } \\
\text { qué pasó. }\end{array}$ & 5.22 & 8.75 & 3.53 \\
\hline 71 & SUP & $\begin{array}{l}\text { Sigue al niño o } \\
\text { se mueve a una } \\
\text { mejor posición } \\
\text { para supervisar o } \\
\text { monitorear cómo } \\
\text { el niño se mueve de } \\
\text { un lugar a otro. }\end{array}$ & 4.69 & 8.25 & 3.56 \\
\hline 62 & -- & $\begin{array}{l}\mathrm{Si} \text { el niño está } \\
\text { molesto o llorando } \\
\text { debido a un } \\
\text { accidente, lo alza } \\
\text { hasta que se calme } \\
\text { y esté listo para } \\
\text { bajarlo. }\end{array}$ & 4.91 & 8.75 & 3.84 \\
\hline
\end{tabular}

Nota $:$ Dim = Dimensión a la que pertenece el ítem; Ptje Medio = Puntaje promedio de las 16 figuras cuidadoras participantes; Ptje Criterio $=$ Puntaje del criterio de la escala; Discr $=$ Discrepancia entre el Ptje Medio y el Ptje Criterio; ABS = Dimensión de Apoyo a la base segura; SUP = Dimensión de Supervisión y monitoreo.

Los guiones -- en la columna de Dim indican que el ítem en cuestión no corresponde a ninguna de las dimensiones.

Tabla 3

Correlación entre seguridad del apego y sus dimensiones

\begin{tabular}{lccccc}
\hline & Seguridad & CIM & PCM & BPM & IOA \\
\hline Seguridad & - & & & & \\
CIM & $.76^{* *}$ & - & & & \\
PCM & $.59^{*}$ & .22 & - & & \\
BPM & .49 & .02 & .38 & - & \\
IOA & .17 & -.15 & .08 & .07 & -
\end{tabular}

Nota $:$ CIM $=$ Calidez en las interacciones con la madre; $\mathrm{PCM}=$ Placer en el contacto físico con la madre; BPM = Búsqueda de proximidad de la madre; IOA = Interacción con otros adultos. $* p<.05 ; * * p<.01$ 
Tabla 4

Discrepancias mayores $a+-3$ puntos en $A Q S(N=16)$

\begin{tabular}{|c|c|c|c|c|c|}
\hline $\begin{array}{l}\text { Ítem } \\
\mathrm{N}^{\circ}\end{array}$ & Dim & Enunciado & Ptje Medio & $\begin{array}{c}\text { Ptje } \\
\text { Criterio }\end{array}$ & Discr \\
\hline 69 & BPM & Rara vez le pide ayuda a la madre. & 2.3 & 6.63 & -4.33 \\
\hline 59 & BPM & $\begin{array}{l}\text { Cuando termina una actividad o termina de jugar, generalmente } \\
\text { encuentra algo más que hacer in regresar a la madre entre actividad. }\end{array}$ & 3.8 & 7.56 & -3.76 \\
\hline 7 & IOA & El niño se ríe y sonríe fácilmente con varias personas. & 4.3 & 7.5 & -3.2 \\
\hline 37 & -- & $\begin{array}{l}\text { El niño es bien activo. Siempre se está moviendo. Prefiere juegos } \\
\text { activos a juegos calmados. }\end{array}$ & 4.8 & 7.9 & -3.1 \\
\hline 68 & -- & En promedio, el niño es una persona más activa que la madre. & 5 & 8.03 & -3.03 \\
\hline 53 & PCM & $\begin{array}{l}\text { Cuando la madre lo alza, el niño pone sus brazos alrededor de ella o } \\
\text { en sus hombros. }\end{array}$ & 8.5 & 5.22 & 3.28 \\
\hline 11 & BPM & $\begin{array}{l}\text { Frecuentemente abraza o busca contacto con la madre sin que ella le } \\
\text { pida o le invite a hacerlo. }\end{array}$ & 7.5 & 3.97 & 3.53 \\
\hline 71 & PCM & $\begin{array}{l}\text { Si la madre lo alza cuando está asustado o molesto, el niño deja de } \\
\text { llorar y se recupera rápidamente. }\end{array}$ & 8.8 & 4.97 & 3.83 \\
\hline 83 & BPM & $\begin{array}{l}\text { Cuando el niño está aburrido va donde la madre buscando algo que } \\
\text { hacer. }\end{array}$ & 6.5 & 2.66 & 3.84 \\
\hline 21 & BPM & $\begin{array}{l}\text { Cuando el niño juega en la casa, está pendiente de la localización de } \\
\text { su madre. La llama de vez en cuando, pone atención cuando cambia de } \\
\text { sitio o de actividad. }\end{array}$ & 8.8 & 4.03 & 4.77 \\
\hline 90 & BPM & $\begin{array}{l}\text { Si la madre se va muy lejos, el niño la sigue y continúa jugando en el } \\
\text { nuevo sitio donde ella está. }\end{array}$ & 8.3 & 3.53 & 4.77 \\
\hline
\end{tabular}

Nota $:$ Dim = Dimensión a la que pertenece el ítem; Ptje Medio = Puntaje promedio de los 16 niños participantes; Ptje Criterio = Puntaje del criterio de la escala; Discr = Discrepancia entre el Ptje Medio y el Ptje Criterio; BPM = Dimensión de Búsqueda de la proximidad de la madre; IOA = Dimensión de Interacción con otros adultos; PCM = Dimensión de Placer en el contacto físico con la madre.

Los guiones -- en la columna de Dim indican que el ítem en cuestión no corresponde a ninguna de las dimensiones.

dimensiones) recibieron un puntaje menor que el criterio de la escala, en tanto que los otros seis ítems (dos de Placer en el contacto físico con la madre y cuatro de Búsqueda de la proximidad de la madre) recibieron un puntaje mayor. El análisis de estos ítems permite deducir que el grupo de niños participantes se muestra más tímido, más dependiente de la madre y con más búsqueda de su proximidad y contacto que lo normado en la escala, lo cual podría estar relacionado con factores culturales.

Respecto del objetivo central de este estudio, se analizó el rol predictor de la sensibilidad de las figuras cuidadoras sobre la seguridad de los niños, considerando también a las variables sociodemográficas de edad e instrucción de las figuras cuidadoras, y de edad y género de los niños. Al introducir simultáneamente todas las variables para el cálculo de regresión lineal múltiple, únicamente la sensibilidad permaneció como predictora en el modelo a un nivel significativo $(F(1,14)=12.73, p=.003)$, en tanto que las variables sociodemográficas fueron excluidas. La relación encontrada entre sensibilidad y seguridad $(r=.690, r 2=.439, p=.003)$, indica que están di- recta, positiva y significativamente correlacionadas a un nivel más alto que el reportado en otros estudios con población latinoamericana (e.g., Posada et al., 2016), y que el $44 \%$ de la variabilidad de la seguridad del apego de los niños puede ser explicada por la sensibilidad de sus figuras cuidadoras.

En cuanto a la correlación entre la sensibilidad y las cuatro dimensiones de la seguridad del apego, se halló que la conducta de la figura cuidadora está significativamente asociada con la calidez del niño en sus interacciones con ella $(r=.59, p=.02)$, y con la búsqueda de su proximidad $(r=49, p=.05)$; sin embargo, no se encontraron relaciones significativas ni marginalmente significativas con el placer que experimenta el niño en el contacto físico con su figura cuidadora $(r=36, p=.17)$, ni con las interacciones que establece con otros adultos $(r=-.29, p=.29)$. Por otro lado, se halló que la seguridad del apego de los niños está correlacionada significativamente con tres de las cuatro dimensiones de la sensibilidad de la figura cuidadora: contribución a interacciones armoniosas $(r=.68, p=.004)$, apoyo a la base segura $(r=.63, p=.01)$, y supervisión y monitoreo 
$(r=.57, p=.02)$. Se encontró que la seguridad del apego de los niños está marginalmente asociada con el establecimiento de límites por parte de su figura cuidadora $(r=.46, p=.08)$.

\section{Discusión}

El presente estudio se fundamentó en la hipótesis de sensibilidad de la teoría del apego, que relaciona la crianza sensible con la seguridad del apego (van IJzendoorn \& Sagi-Schwartz, 2008). Con la finalidad de generar conocimiento contextual y disponer de datos referenciales que sirvan de base para futuros estudios, se trabajó con 16 figuras cuidadoras y sus hijos preescolares de desarrollo típico, residentes en Quito, Ecuador, contexto en el cual no se registran investigaciones sobre el tema. El objetivo fue analizar, en la población estudiada, la relación entre sensibilidad y seguridad.

Respecto de las características sociodemográficas de los participantes, se encontraron dos datos de interés. Por un lado, se halló que en el $100 \%$ de los casos, las principales figuras cuidadoras de los niños eran las madres, quienes ven al padre como ayuda en la crianza de los hijos y se perciben a sí mismas como las principales responsables; no obstante, la mayoría de ellas poseen una preparación académica que les permite desenvolverse en el ámbito laboral (en esta investigación el $81 \%$ tiene educación superior: $62 \%$ de pregrado y $19 \%$ de posgrado). Por otro lado, se constató que el $50 \%$ de los niños eran hijos únicos, lo cual refleja una tendencia en la constitución actual de la familia ecuatoriana que vive en las ciudades.

En cuanto a la sensibilidad de las figuras cuidadoras, se encontró entre las participantes un índice promedio de .43 , que es semejante al reportado en otros estudios con población latina (e.g., Posada et al., 1999; Posada et al., 2016), específicamente, al hallado en México (.46) y en Colombia (.48) (Posada et al., 2016). Se encontró también que la sensibilidad de las figuras cuidadoras está relacionada con su edad, lo que permite concluir que la madurez se asocia positivamente con conducta sensible; este hallazgo ha sido previamente reportado por una variedad de investigaciones (e.g., Bornstein, Hendricks, Haynes, \& Painter, 2007; Demers, Bernier, Tarabulsy, \& Provost, 2010; Posada et al., 2016; Santelices et al., 2015). Adicionalmente, se halló que las di- ferentes dimensiones de la conducta sensible de las participantes se encuentran relacionadas entre sí, lo que permite inferir que han desarrollado su sensibilidad con la misma intensidad en los diferentes dominios.

Además, de manera general se observa que las figuras cuidadoras que contribuyen al establecimiento de interacciones armoniosas, que apoyan a los niños en el establecimiento de una base segura, que los supervisan, monitorean y establecen límites de forma sensible, tienen hijos que han desarrollado un vínculo más seguro con ellas. A pesar de ello, llama la atención la alta dispersión de algunas puntuaciones que en algunos casos alcanzan valores negativos; aunque en sentido estricto, aquello indicaría poca sensibilidad, es importante considerar que las prácticas de crianza cambian de un contexto a otro (e.g., Ke1ler, 2013; Quinn \& Mageo, 2013), y que las variaciones intraculturales suelen ser incluso mayores que las interculturales (Mesman et al., 2016; van IJzendoorn \& Kroonenberg, 1998), lo cual puede ser particularmente evidente en países pluriculturales como el Ecuador (Villamarín, 2017). De hecho, el puntaje de los ítems mostrados en la Tabla 2 indican por un lado, que las puntuaciones extremas al ser promediadas, se sitúan en la media, pero por otro lado también muestran una tendencia general de las figuras cuidadoras a ubicarse en un punto medio entre proteger al niño y dejar que él se enfrente a las situaciones con sus propios recursos, lo cual puede ser diferente de lo normado, pero no por ello menos sensible.

En referencia a la seguridad del apego, los niños participantes en este estudio, como grupo, alcanzaron puntajes superiores (.42) a los de otros grupos de niños latinoamericanos de condiciones sociodemográficas semejantes (Nóblega, 2012; Posada et al., 2013; Posada et al., 2016). Además, todos obtuvieron puntuaciones positivas, lo cual indica que, en términos generales, han desarrollado un nivel adecuado de seguridad en sus relaciones de apego. No obstante, a diferencia de la conducta materna, los niños estudiados no presentan una alta consistencia en sus conductas; si bien se encontró una elevada asociación con calidez en las interacciones con la madre, la relación entre seguridad del apego, placer en el contacto físico y búsqueda de proximidad con ella es apenas moderada.

Adicionalmente, los resultados también confirmaron un dato reportado con anterioridad en 
población peruana, en relación a la relativa independencia entre la naturaleza de la interacción que establece el niño con otros adultos y su nivel de seguridad global (Nóblega, 2012). Frente a este resultado, los aspectos culturales han sido considerados como una posible explicación, en el sentido de que en los contextos latinos se otorga una alta importancia al respeto y al cumplimiento de ciertas normas de comportamiento por parte de los niños hacia las otras personas (Nóblega, 2012). De hecho, los puntajes elevados de los niños participantes en varios ítems de búsqueda de la proximidad de la madre estarían confirmando este planteamiento en el sentido de que, ante la presencia de personas nuevas, buscan refugio en la figura cuidadora.

En relación con el objetivo central, se encontró a la sensibilidad como variable predictora a nivel significativo de la seguridad del apego, con una relación entre ellas de .69, que es mayor a la hallada en los meta análisis realizados internacionalmente (e.g., De Wolff \& van IJzendoorn, 1997; Atkinson et al., 2000) y en los estudios en contextos latinos en los que se reporta un rango entre .30 en México y .43 en Perú (Posada et al., 2016).

\section{Conclusiones}

En síntesis, los resultados del presente estudio permiten concluir que las figuras cuidadoras participantes demuestran un nivel de sensibilidad hacia las necesidades de sus hijos preescolares, similar al encontrado en otros estudios latinoamericanos; se halló, además, asociación entre edad de la madre y conducta sensible y correlación alta entre las cuatro dimensiones de la sensibilidad. Un dato de interés que se observó en la interacción es que las figuras cuidadoras, en general, dejan que los niños enfrenten situaciones con sus propios recursos, lo cual parece responder a una práctica de crianza.

En cuanto a la seguridad del apego de los niños, se encontró un nivel ligeramente más elevado que los reportados en otros estudios latinoamericanos; adicionalmente, se halló correlación alta entre seguridad y calidez en las interacciones con la madre, correlación moderada con placer en el contacto físico con ella y con búsqueda de su proximidad, relativa independencia entre seguridad e interacción con otros adultos, posible- mente por el respeto y la distancia que caracteriza la relación de los niños con las personas mayores en los contextos latinos, como han planteado estudios previos.

Del mismo modo que en otras investigaciones, en este trabajo también se encontró a la sensibilidad como predictora de la seguridad del apego; se hallaron, además, asociaciones entre la sensibilidad de la figura cuidadora y la calidez y búsqueda por parte del niño de su proximidad, y entre la seguridad del niño y la contribución a interacciones armoniosas por parte de la madre. Al ser Ecuador un país diverso y pluricultural, estos resultados pueden servir de base para investigaciones más profundas en las que se incluya a la figura paterna, a niños de otros contextos culturales y de otras condiciones de desarrollo, de modo que se logre un conocimiento mayor del tema.

Es importante mencionar como limitaciones de este estudio, el reducido número de participantes y de visitas realizadas a cada díada. Además, es necesario recalcar que algunos aspectos metodológicos pueden haber favorecido los resultados, tales como la edad de los niños, la naturaleza de los métodos de recolección de información, así como la concurrencia temporal en la medición de la conducta materna e infantil, factores que han sido reportados en la literatura como influyentes en el aumento de los valores de las correlaciones (e.g., Atkinson et al., 2000; De Wolff \& van IJzendoorn, 1997; Nóblega, 2012).

Queremos expresar nuestro agradecimiento a la Pontificia Universidad Católica del Ecuador (PUCE), la institución que financió esta investigación en su totalidad, y al trabajo de las estudiantes de Psicología Educativa de la PUCE, María José Barreiro y María José Cuesta, quienes participó como asistentes de investigación.

\section{Referencias}

Ainsworth, M. D. (1967). Infancy in Uganda: Infant care and the growth of love. Baltimore: Johns Hopkins University.

Ainsworth, M. D. (1969). Maternal Sensitivity Scales. The Baltimore Longitudinal Project. Recuperado de http:// www.psychology.sunysb.edu/attachment/measures/ content $/$ maternal $\% 20$ sensitivity $\% 20$ scales.pdf

Ainsworth, M. D., Blehar, M. C., Waters, E., \& Wall, S. (1978; 2015). Patterns of attachment: A psychological study of the Strange Situation. Bulgay, Suffolk, UK: Taylor \& Francis. 
Atkinson, L., Niccols, A., Paglia, A., Coolbear, J., Parker, K., Poulton, K., . . . Sitarenios, G. (2000). A Metaanalysis of time between maternal sensitivity and attachment assesments: Implications for internal working models in infancy/toddlerhood. Journal of Social and Personal Relationships, 17, 791-810. doi:10.1177/0265407500176005

Bakermans-Kranenburg, M. J., \& van Ijzendoorn, M. H. (2016). Attachment, Parenting and Genetics. In J. Cassidy, \& P. R. Shaver (Eds.), Handbook of Attachment: Theory, Research, and Clinical Applications (3 ed., pp. 155-179). New York: The Guilford Press.

Bornstein, M. H., Hendricks, C., Haynes, O. M., \& Painter, K. M. (2007). Maternal sensitivity and child responsiveness: Associations with social context, maternal characteristics, and chid characteristics in a multivariate analysis. Infancy, 12(2), 189-223. doi:10.111/j.1532-7078.2007.tb00240.x

Bowlby, J. (1969/1998). El Apego y la Pérdida: El Apego (Vol. 1). Barcelona: Ediciones Paidós Ibérica.

Bowlby, J. (1973). Attachment and loss - Vol. 2. Separation: Anxiety and anger. New York: Basic Books.

Bowlby, J. (1988). Una base segura. Buenos Aires: Paidós.

Carbonell, O. A. (2013). Conferencia: La sensibilidad del cuidador y su importancia para promover un cuidado de calidad en la primera infancia. Ciencias Psicológicas, 7(2), 201-207.

Cerezo, M. Á., Pons-Salvador, G., \& Trenado, R. (2011). La cualidad del apego infantil y sensibilidad materna desde la perspectiva microsocial. Acción Psicológica, $8(2), 9-25$.

Coan, J. A. (2016). Toward a Neuroscience of Attachment. In J. Cassidy, \& P. R. Shaver (Eds.), Handbook of Attachment: Theory, Research, and Clinical Applications (3 ed., pp. 242 - 269). New York: The Guilford Press.

De Wolff, M. S., \& van Ijzendoorn, M. H. (1997). Sensitivity and Attachment: A Meta-Analysis of Parental Antecedents of Infant Attachment. Child Development, 68(4), 571-591.

Demmers, I., Bernier, A., Tarabulsy, G. M., \& Provost, M. A. (2010). Mind-mindedness in adult and adolescent mothers: Relations to maternal sensitivity and infant attachment. International Journal of Behavioral Development, 34(6), 529-537. doi: $10.1177 / 0165025410365802$

Guerrero, X. (2013). Estudio del tipo de personalidad de las madres que acuden al Centro Materno Infantil Ambato $N^{\circ} 2$ y su relación con el tipo de apego que generan con sus hijos. Trabajo de Grado previo a la obtención del título de Magíster en Psicoterapia Integrativa. Quito, Ecuador: Universidad Central del Ecuador, Facultad de Ciencias Psicológicas.

Keller, H. (2013). Atacchment and Culture. Journal of Cross-Cultural Psychology, 44(2), 175-194. doi: $10.1177 / 0022022112472253$

Lecannelier, F., Kimelman, M., González, L., Núñez, C., \& Hoffmann, M. (2008). Evaluación de Patrones de Apego en Infantes durante su Segundo Año en dos Centros de Atención de Santiago de Chile. Revista Argentina de Clínica Psicológica, XVII, 197-207.

Marvin, R. S., Britner, P. A., \& Russell, B. S. (2016). Normative Development: The Ontogeny of Attachment in Childhood. In J. Cassidy, \& P. R. Shaver (Eds.), Handbook of Attachment: Theory, Research, and Clinical Applications (3 ed., pp. 273 - 290). New York: The Guilford Press.
Mesman, J., \& Emmen, R. A. (2013). Mary Ainsworth's legacy: a sistematic review of observational instruments measuring parental sensitivity. Attachment \& Human Development, 15(5-6), 485-506. doi:10.1080/1461673 4.2013.820900

Mesman, J., van IJzendoorn, M. H., \& Sagi-Schwartz, A. (2016). Cross-Cultural Patterns of Attachment: Universal and Contextual Dimensions. In J. Cassidy, \& P. R. Shaver (Eds.), Handbook of Attachment: Theory, Research and Clinical Applications (3 ed., pp. 852877). New York: The Guilford Press.

Nóblega, M. (2012). Conducta de base segura y sensitividad en niños y madres del Distrito Los Olivos. Tesis para optar el Grado de Doctora en Psicología. Lima, Perú: Pontificia Universidad Católica del Perú.

Nóblega, M., Bárrig, P., Conde, G., Núñez del Prado, J., Carbonell, O. A., Altmann de Litvan, M., . . . Bauer, M. (2016). Cuidado materno y seguridad del apego antes del primer año de vida. Universitas Psychologica, 15(1), 245-260.

Orbe, C. (2012). El estilo de apego del niño con la madre como factor de influencia en el proceso de adaptación al medio preescolar. Tesis de Maestría. Quito, Ecuador: Universidad San Francisco de Quito.

Orbe, C. (2015). La Teoría del Apego desde la visión Mente - Cerebro - Educación. Revista PUCE, (100), 237-258.

Pederson, D. R., Bailey, H. N., Tarabulsy, G. M., Bento, S., \& Moran, G. (2014). Understanding sensitivity: Lessons learned from the legacy of Mary Ainsworth. Attachment and Human Development, 16(3), 261-270. doi:10.1080/14616734.2013.842753

Posada, G. (2016). Piecing together the sensitivity construct: ethology and cross cultural research. In K. E. Grossman, I. Bretherton, E. Waters, \& K. Grossman (Eds.), Maternal Sensitiviy: Mary Ainsworth's Enduring Influence on Attachment Theory, Research, and Clinical Applications. New York, US: Taylor \& Francis.

Posada, G., Carbonell, O. A., Alzate, G., \& Plata, S. J. (2004). Through Colombian Lenses: Ethnographic and Conventional Analyses of Maternal Care and Their Associations With Secure Base Behavior. Developmental Psychology, 40(4), 508 - 518. doi:10.1037/0012-1649.40.4.508

Posada, G., Jacobs, A., Carbonell, O. A., Alzate, G., Bustamante, M., \& Arenas, A. (1999). Maternal care and attachment security in ordinary contexts. Developmental Psychology, 30(6), 1379 - 1388. doi:10.1037/00121649.35.6.1379

Posada, G., Jacobs, A., Richmond, M., Carbonell, O., Alzate, G., Bustamante, M. R., \& Quiceno, J. (2002). Maternal Caregiving and Infant Security in Two Cultures. Developmental Psychology, 38(1), 67-78. doi:10.1037//0012-1649.38.1.67

Posada, G., Kaloustian, G., Richmond, M. K., \& Moreno, A. (2007). Maternal secure base support and preschoolers' secure base behavior in natural environments. Attachment \& Human Development, 9(4), 393 - 411. doi:10.1080/14616730701712316

Posada, G., Trudel, M., Plata, S. J., Peña, P. P., Pérez, J., Tereno, S., . . . Keng-Ling, L. (2013). Is the Secure Base Phenomenon Evident Here, There, and Anywhere? A Cross-Cultural Study of Child Behavior and Experts' Definitions. Child Deveopment, 84(6), 18961905. doi:10.1111/cdev.12084

Posada, G., Trumbell, J., Nóblega, M., Plata, S., Peña, P., Carbonell, O., \& Lu, T. (2016). Maternal Sensitivity and Child Secure Base Use in Early Childhood: Studies in Differen Cultural Contexts. Child Devolpment, 87(1), 297-311. doi:10.1111/cdev.12454 
Posada, G., Waters, E., Crowell, J., \& Lay, K. L. (1995). Is it easier to use a secure mother as a secure base? Attachment Q-set correlates of the adult attachment interview. (E. Waters, B. Vaughn, G. Posada, \& K. Kondo-Ikemura, Eds.) Monographs of the Society for Research in Child Development, 60(2-3), 133 - 145. doi:10.2307/1166175

Quinn, N., \& Mageo, J. M. (2013). Attachment Reconsidered: Cultural Perspectives on a Western Theory. New York: NY Palgrave Macmillan.

Richmond, M., Posada, G., \& Jacobs, A. (2001). Maternal behavior and attachment security in 3 year-olds: A naturalistic study. Poster presented at the meeting of the Society for Research in Child Development. Minneapolis, MN.

Salinas-Quiroz, F., \& Posada, G. (2015). MBQS: Método de evaluación para intervenciones en apego dirigidas a la primera infancia. Revista Latinoamericana de Ciencias Sociales, Niñez y Juventud, 13(2), 1051-1063. doi :10.11600/1692715x.13235280514

Salinas-Quiroz, F., Morales-Carmona, F. A., de Castro, F., Juárez-Hernández, M. C., Posada, G., \& Carbonell, O. A. (2015). Educación Inicial de Base Segura: Indicador de la calidad educativa para la primera infancia. Psicología Iberoamericana, 23(1), 75-82.

Santelices, M. P., Farkas, C., Montoya, M. F., Galleguillos, F., Carvacho, C., Fernández, A., . . . Himmel, É. (2015). Factores predictivos de sensibilidad materna en infancia temprana. Psicoperspectivas, 14(1), 6676. doi:10.5027/PSICOPERSPECTIVAS-VOL14ISSUE-FULLTEXT-441

Schore, A. N. (2017). Modern Attachment Theory. In S. N. Gold (Ed.), APA Handbook of Trauma Psychology: Vol 1. Foundations in Knowledge (pp. 389-406). American Psychological Association. doi:10.1037/0000019-020

Simpson, J. A., \& Belsky, J. (2016). Attachment Theory within Modern Evolutionary Framework. In J. Cassidy, \& P. R. Shaver (Eds.), Handbook of Attachment: Theory, Research, and Clinical Apllications (3 ed., pp. 91 - 116). New York: The Guilford Press.
Solomon, J., \& George, C. (2016). The Measurement of Attachment Security and Related Constructs in Infancy and Early Childhood. In J. Cassidy, \& P. R. Shaver (Eds.), Handbook of Attachment: Theory, Research, and Clinical Applications (3 ed., pp. 366 - 396). New York: The Guilford Press.

van Ijzendoorn, M. H., \& Kroonenberg, P. M. (1988). Crosscultural patterns of attachment: A meta-analysis of the Strange Situation. Child Development, 59, 147-156.

van Ijzendoorn, M. H., \& Sagi-Schwartz, A. (2008). Crosscultural patterns of attachment: Universal and contextual dimensions. In J. Cassidy, \& P. R. Shaver (Eds.), Handbook of Attachment: Theory, Research and Clinicial Applications (2 ed., pp. 880-905). New York. US: The Guilford Press.

van Ijzendoorn, M. H., Vereijken, C. M., BakermansKranenburg, M. J., \& Riksen-Walraven, J. M. (2004). Assesing attachment security with the Attachment Q-sort: Meta-analytic evidence for the validity of the observer AQS. Child Development, 75, 1188-1213. doi:10.1111/j.1467-8624.2004.00733.x

Vaughn, B. E., Coppola, G., Verissimo, M., Monteiro, L., Santos, A. J., Posada, G., . . Korth, B. (2007). The quality of maternal secure-base scripts predicts children's secure-base behavior at home in three sociocultural groups. International Journal of Behavioral Development, 31(1), 65-76. doi:10.1177/0165025407073574

Villamarín, G. (2017). Consideraciones culturales sobre la práctica de la Psicología: el caso de la Teoría del Apego. Programa de Maestría en Estudios de la Cultura. Quito: Universidad Andina Simón Bolívar, sede Ecuador.

Waters, E. (1995). Appendix A: The Attachment Q Set. (E. Waters, B. Vaughn, G. Posada, \& K. Kondo-Ikemura, Eds.) Monographs of the Society for Research in Child Development, 60(2-3), 234 - 246. doi:10.2307/116618 